\title{
Identifying the Genes Responsible for Iron-Limited Condition in Riemerella anatipestifer CH-1 through RNA-Seq-Based Analysis
}

\author{
MaFeng Liu, ${ }^{1,2,3}$ Mi Huang, ${ }^{1,2,3}$ DeKang Zhu, ${ }^{2,3}$ MingShu Wang, ${ }^{1,2,3}$ \\ RenYong Jia, ${ }^{1,2,3}$ Shun Chen, ${ }^{1,2,3}$ KunFeng Sun, ${ }^{1,2,3}$ Qiao Yang, ${ }^{1,2,3}$ Ying Wu, $^{1,2,3}$ \\ Francis Biville, ${ }^{4}$ and AnChun Cheng ${ }^{1,2,3}$ \\ ${ }^{1}$ Institute of Preventive Veterinary Medicine, Sichuan Agricultural University, Chengdu, Sichuan 611130, China \\ ${ }^{2}$ Research Center of Avian Disease, College of Veterinary Medicine of Sichuan Agricultural University, Chengdu, Sichuan 611130, China \\ ${ }^{3}$ Key Laboratory of Animal Disease and Human Health of Sichuan Province, Chengdu, Sichuan 611130, China \\ ${ }^{4}$ Unité des Infections Bactériennes Invasives, Département Infection et Epidémiologie, Institut Pasteur, Paris, France
}

Correspondence should be addressed to MaFeng Liu; liumafengra@163.com and AnChun Cheng; chenganchun@vip.163.com

Received 28 December 2016; Accepted 29 March 2017; Published 30 April 2017

Academic Editor: Ernesto Picardi

Copyright (C) 2017 MaFeng Liu et al. This is an open access article distributed under the Creative Commons Attribution License, which permits unrestricted use, distribution, and reproduction in any medium, provided the original work is properly cited.

\begin{abstract}
One of the important elements for most bacterial growth is iron, the bioavailability of which is limited in hosts. Riemerella anatipestifer ( $R$. anatipestifer, RA), an important duck pathogen, requires iron to live. However, the genes involved in iron metabolism and the mechanisms of iron transport are largely unknown. Here, we investigated the transcriptomic effects of iron limitation condition on $R$. anatipestifer CH-1 using the RNA-Seq and RNA-Seq-based analysis. Data analysis revealed genes encoding functions related to iron homeostasis, including a number of putative TonB-dependent receptor systems, a HmuY-like protein-dependent hemin (an iron-containing porphyrin) uptake system, a Feo system, a gene cluster related to starch utilization, and genes encoding hypothetical proteins that were significantly upregulated in response to iron limitation. Compared to the number of upregulated genes, more genes were significantly downregulated in response to iron limitation. The downregulated genes mainly encoded a number of outer membrane receptors, DNA-binding proteins, phage-related proteins, and many hypothetical proteins. This information suggested that RNA-Seq-based analysis in iron-limited medium is an effective and fast method for identifying genes involved in iron uptake in $R$. anatipestifer $\mathrm{CH}-1$.
\end{abstract}

\section{Introduction}

Riemerella anatipestifer ( $R$. anatipestifer, RA) is a Gramnegative bacterium that belongs to the family Flavobacteriaceae in the rRNA superfamily $\mathrm{V}$ [1]. R. anatipestifer infection causes disease in ducks, geese, chickens, turkeys, and other waterfowl and birds [2]. The disease presents as an acute or chronic septicemia characterized by meningitis, fibrinous pericarditis, perihepatitis, and other symptoms [3]. The disease causes increased mortality and decreased weight and is estimated to result in huge economic losses to the duck industry each year worldwide. At present, at least 21 serotypes of $R$. anatipestifer have been identified in the world $[2,4]$.

Iron is one of the most important elements for bacterial growth, as it is an essential cofactor in many important enzymes involved in energy metabolism and nucleotide synthesis [5]. Iron is the second most abundant metal on earth, but it exists primarily in the insoluble ferric oxide form under aerobic conditions, which is not available for bacterial growth [5]. Inside the host, most iron is bound to ironbinding proteins, such as ferritin, transferrin, and lactoferrin. Iron could also be included in the heme of hemoproteins (the terminology "heme" was used when talking about hemoproteins) [6]. Bacteria employ various mechanisms to capture iron from the outside [7]. One of these mechanisms is the secretion of a small molecular compound, a siderophore, which sequesters iron from the outside environment by highaffinity interactions [8]. Then, iron-bound siderophores are taken up by the bacteria through specific siderophore receptors and transport systems [9]. Alternatively, some pathogens 
have specific cell surface receptors that bind hemin (the terminology "hemin" was used when talking about iron and protoporphyrin ring source) or hemoprotein and transport hemin to the cell or secreted hemophores that capture hemin from host hemoproteins and then deliver hemin to bacterial surface receptors [10]. Therefore, iron-limited conditions are able to prompt most bacteria to upregulate the expression of genes related to iron/hemin uptake, such as iron/hemin transporters and siderophore biosynthetic enzymes [11-13].

$R$. anatipestifer requires iron and hemin to survive [14]. Genome analysis has shown that $R$. anatipestifer codes for a large number of TonB-dependent receptors, a TonB family protein, two sets of TonB complexes, and an FeoAB system [15]. In a previous study, we demonstrated that TonB1 and TonB2 are involved in hemin uptake by $R$. anatipestifer ATCC11845 [14]. Moreover some hemin binding proteins were detected in $R$. anatipestifer $\mathrm{CH}-1$ [16]. However, other genes involved in iron/hemin uptake by $R$. anatipestifer are largely unknown. In this study, we analyzed the global transcriptomic changes in $\mathrm{R}$. anatipestifer $\mathrm{CH}-1$ under ironlimited conditions. Here, we observed wide-ranging effects on the transcripts of iron-related genes of $R$. anatipestifer $\mathrm{CH}-1$ and identified some new genes involved in iron/hemin uptake.

\section{Materials and Methods}

2.1. Bacterial Strains and Growth Conditions. For transcriptome analyses, $R$. anatipestifer $\mathrm{CH}-1$ was grown in tryptone soy broth (TSB) medium (Sigma, China) as the iron-replete condition, while the iron-limited condition was TSB supplemented with $100 \mu \mathrm{M}$ iron chelator $2,2^{\prime}$-dipyridyl (Dip). The bacteria were cultured at $37^{\circ} \mathrm{C}$ with shaking at $180 \mathrm{rpm} / \mathrm{min}$. Then, they were harvested at OD600 $=0.6$ for iron-limited cultures and OD600 $=1.1$ for iron-replete cultures (Figure 1).

2.2. RNA-Seq. Total RNA extraction was performed using the RNeasy Protect Bacteria Mini Kit (QIAGEN, Cat. number 74524) using the protocol described by Liu et al. [17]. A total amount of $3 \mu \mathrm{g}$ RNA per sample was used for the RNA sample preparations. RNA quantification, library preparation, and sequencing were performed at Beijing Novogene as described elsewhere. Then the clean data were obtained by removing reads containing adapter, reads containing ploy$\mathrm{N}$, and low-quality reads from raw data [18]. The highquality reads obtained for each library were shown in Table 1. Then the $R$. anatipestifer $\mathrm{CH}-1$ genome (CP003787.1) and gene model annotation files were downloaded from genome website (https://www.ncbi.nlm.nih.gov/nuccore/CP003787.1) directly, using Bowtie2-2.2.3 to build index and align clean reads of the $R$. anatipestifer $\mathrm{CH}-1$ genome [19].

2.3. Real-Time PCR Validation of RNA-Seq. The differential expression of selected genes was validated by quantitative reverse transcription polymerase chain reaction (qRTPCR) using the SYBR green-based detection system on a CFX Connect ${ }^{\circledR}$ Real-Time PCR Detection System (BioRad Laboratories, Hercules, CA) using the KAPA SYBR ${ }^{\circledR}$

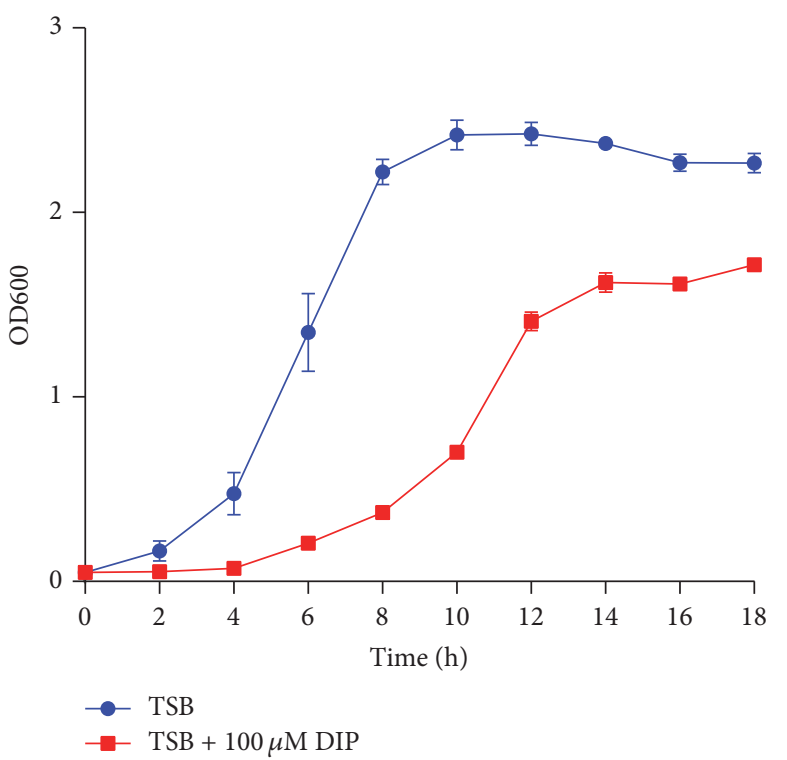

Figure 1: Growth curves of $R$. anatipestifer $\mathrm{CH}-1$ in iron-limited and iron-replete media. Optical densities at a wavelength of $600 \mathrm{~nm}$ were taken from the 2 nd to the 18th hours at intervals of 2 hours. Measurements were performed on triplicate samples.

FAST qPCR kit (KAPABIOSYSTEMS, Boston, USA). cDNA was synthesized from each RNA sample $(1 \mu \mathrm{g})$ using the HiScript ${ }^{\mathrm{TM}}$ Q RT SuperMix for qPCR (+gDNA wiper) (R12301; Vazyme, Nanjing, China). Real-time PCR assays were conducted with the primers for real-time PCR listed in Table S1 in Supplementary Material, available online at https://doi.org/10.1155/2017/8682057. Quantitative PCR was performed on samples deposited in triplicate using the standard curve mode protocol in which the calibration curve was generated using serial fivefold dilutions of $100 \mathrm{ng}$ of total RNA. The RNA quantity was normalized using a probe specific for $16 \mathrm{~S}$ rRNA.

2.4. RNA-Seq Analysis. To quantify the expression level of genes, HTSeq v0.6.1 was used to count the read numbers mapped to each gene [20]. Then, the FPKM (expected number of Fragments Per Kilobase of transcript sequence per Million base pairs sequenced) of each gene was calculated based on the length of the gene and the read counts mapped to this gene. Prior to differential gene expression analysis, for each sequenced library, the read counts were adjusted by edgeR program package through one scaling normalized factor [21]. In this study, we used the DEGSeq $\mathrm{R}$ package (1.20.0) to execute the differential expression analysis of two conditions [22]. Corrected $P$ value of 0.005 and $\log 2$ (fold change) of 1 were set as the threshold for significantly differential expression. To analyze the gene structure of $R$. anatipestifer CH-1, Rockhopper was used to identify operons and transcription start sites. This program can be used for efficient and accurate analysis of bacterial RNA-Seq data and can aid in the elucidation of bacterial transcriptomes [23]. Moreover we used the aligned paired-end reads to infer the operonic structure of $\mathrm{R}$. anatipestifer $\mathrm{CH}-1$ transcripts. 
TABLE 1: Summary of Illumina RNA-Seq data.

\begin{tabular}{lcccc}
\hline Sample & Total reads & Total mapped & Clean data $^{*}(\mathrm{~Gb})$ & Percentage of sequence reads mapped \\
\hline CH_1TSB & 17948156 & 17106745 & 2.24 & $95.31 \%$ \\
CH_1TSBD & 21489778 & 21099289 & 2.68 & $98.18 \%$ \\
\hline
\end{tabular}

${ }^{*}$ Clean data were obtained from raw data by removing reads containing adapter and poly- $\mathrm{N}$ and low-quality reads.

If genes obtained $>20$ reads aligning on both genes in a sequencing sample, they would be selected as potential in an operonic structure. Sequential genes that are present in operonic structure were merged together to form potential operonic transcripts (Table S2).

\section{Results and Discussion}

3.1. Growth of R. anatipestifer CH-1 in TSB and TSB with Dip. To evaluate the effect of iron restriction on the growth of $R$. anatipestifer $\mathrm{CH}-1$, we grew $R$. anatipestifer $\mathrm{CH}-1$ in TSB and TSB with $100 \mu \mathrm{M}$ Dip, which restricts most iron. Figure 1 showed that the growth of $R$. anatipestifer $\mathrm{CH}-1$ was seriously hindered when iron was restricted, indicating that iron is an essential element for $R$. anatipestifer. Thus, this condition was suitable for performing RNA-Seq.

\subsection{General Assessment of Iron Limitation Transcriptomic} Datasets. Over 95\% of all clean reads aligned to coding regions of the $R$. anatipestifer $\mathrm{CH}-1$ genome (Table 1). Since only one sample in the different iron condition was used to perform RNA-Seq, qRT-PCR validation was performed on the transcriptome data using a subset of 20 differentially regulated genes (Table S1). The transcriptome data generally corresponded well with the qRT-PCR data, with a Pearson correlation coefficient of 0.806 (Figure 2), illustrating that our RNA-Seq data were of suitable quality for transcriptome analysis.

Upon comparing cultures grown in TSB and TSB with Dip, overall differences in gene expression were observed (Figure 3). To examine these differences further, DEGs (differentially expressed genes) were identified using the DEseq package [22]. A total of 463 DEGs were identified, including 80 upregulated (Table 2) and 383 downregulated genes (Table S3). These genes represent $23 \%$ of the genome (2038 genes) [15]. The large number of DEGs suggests that iron-limited environments have global effects on $R$. anatipestifer $\mathrm{CH}-1$. Since samples of different OD were used to perform RNASeq, this study can not exclude the fact that cell density might influence gene expression.

3.3. Genome-Wide Identification of $\mathrm{R}$. anatipestifer $\mathrm{CH}-1$ Genes in Operonic Structures. In addition to identifying gene boundaries, we drew on paired-end sequencing information to identify the $R$. anatipestifer $\mathrm{CH}-1$ global operonic structure. In total, 377 genes were determined to be in operonic structures using this analysis, thus constituting 230 operons (Table S2). These genes represent $18 \%$ of the genome (2038 genes) [15].
3.4. Gene Ontology (GO) Annotation and Kyoto Encyclopedia of Genes and Genomes (KEGG) Pathway Mapping of $D E G s$. The DEGs were assigned to 26 functional groups by enrichment analysis of Gene Ontology (GO) assignments [18]. In the three main GO categories of biological process, cellular component, and molecular function, genes in the role categories of "localization, transport, and establishment of localization" in biological process, "membrane" in cellular component or "receptor activity and transporter activity" in molecular function were notably up- or downregulated (Figure 4).

The biological functions associated with the DEGs were further analyzed in terms of enriched Kyoto Encyclopedia of Genes and Genomes (KEGG) pathways [24], and a total of 20 pathways were predicted (Figure 5). Among these pathways, "microbial metabolism in diverse environments," "ribosome," and "thiamine metabolism" were the most highly represented categories (Figure 5).

\subsection{Iron Limitation Increased the Transcription of Putative} Iron Acquisition Systems. Genome sequence analysis indicated that $R$. anatipestifer $\mathrm{CH}-1$ encodes $\mathrm{Fe}^{2+}$ and $\mathrm{Fe}^{3+}$ acquisition systems [15]. Once in the periplasm, $\mathrm{Fe}^{2+}$ is taken across the inner membrane via a divalent metal uptake system, such as the Feo system of E. coli [25] and the Yfe system of Yersinia pestis [26]. In this study, the predicted genes feoB (B739_0594) and feoA (B739_0595), which encode an $\mathrm{Fe}^{2+}$ transporter, were highly upregulated in the ironlimited condition, suggesting a role in the uptake of ferrous iron (Table 2). Sequence comparison revealed that all of the sequenced $R$. anatipestifer genomes have homologues of FeoA and FeoB of $R$. anatipestifer $\mathrm{CH}-1$, with similarities of $100 \%$ for FeoA and between $88 \%$ and $100 \%$ for FeoB. In turn, these $R$. anatipestifer $\mathrm{CH}-1$ genes have $33.45 \%$ and $32.89 \%$ identities to the FeoA and FeoB products of $E$. coli, respectively. In $E$. coli, this operon is regulated by Fur and is induced in acidic conditions [27]. The functions of FeoA and FeoB and their regulation in $R$. anatipestifer are underinvestigated.

In aerobic conditions, many bacteria produce siderophores to solubilize $\mathrm{Fe}^{3+}$. Then, siderophore-bound $\mathrm{Fe}^{3+}$ is taken up by TonB-dependent receptors [5]. Genome analysis revealed that there are at least 33 predicted TonBdependent receptors in $R$. anatipestifer $\mathrm{CH}-1$, some of which are predicted transporters for ferric-siderophore complexes or heme. In this study, 5 TonB-dependent receptors were upregulated (B739_0094, B739_0103, B739_0173, B739_1068, and B739_1416) in the presence of iron depletion. The 


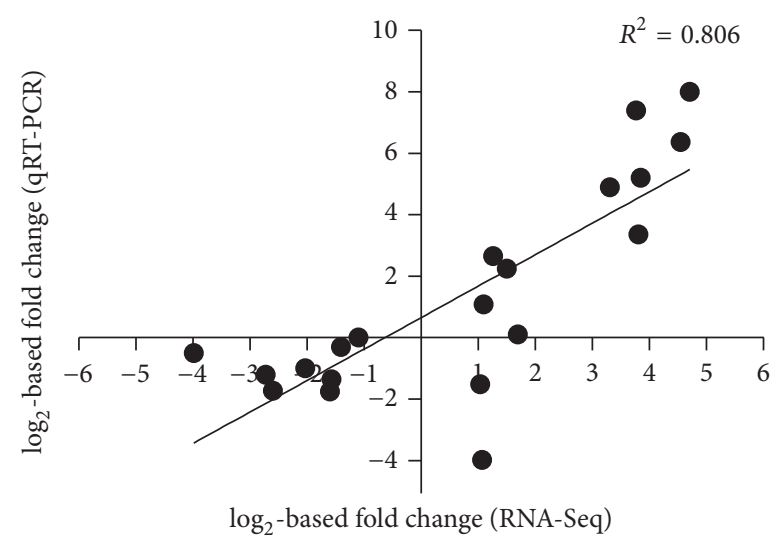

FIGURE 2: Validation of RNA-Seq data. Correlation analysis of $\log _{2}$-based fold changes between RNA-Seq data and qRT-PCR data for 20 genes of $R$. anatipestifer CH-1. The chart depicts a plot of RNA-Seq $\log _{2}$-based fold changes versus qRT-PCR $\log _{2}$-based fold changes for transcripts of genes in cultures of $R$. anatipestifer CH-1 grown in TSB+Dip medium versus TSB medium. A Pearson correlation coefficient of 0.806 was noted.

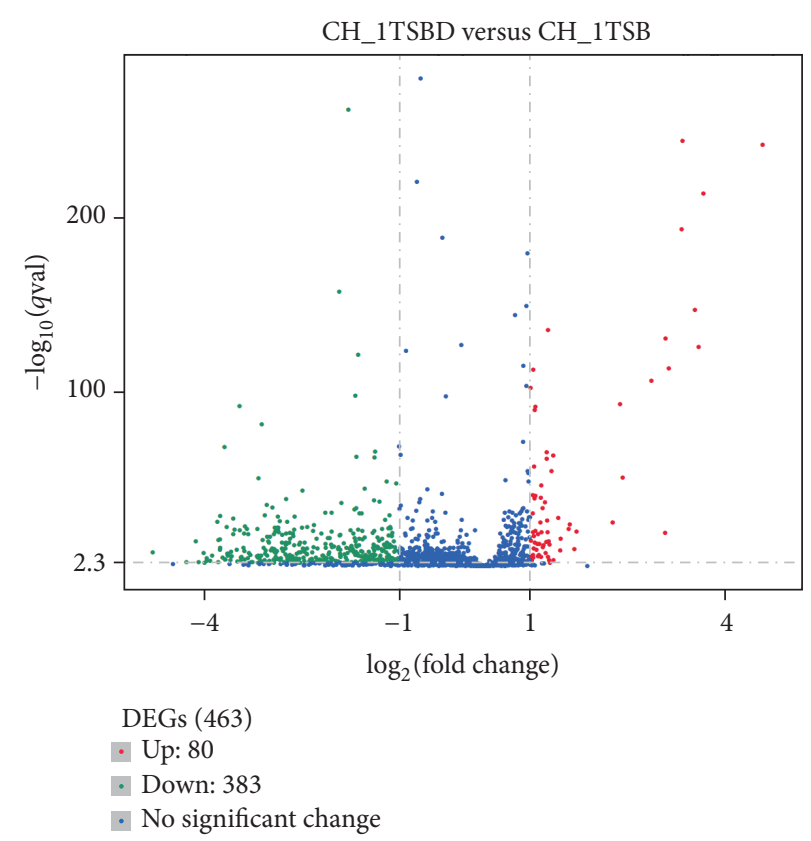

FIGURE 3: Differential gene transcription in cells grown in iron-limited TSB medium compared to TSB medium. The $x$-axis of the chart shows log2-based fold changes of transcripts in cells grown in iron-limited medium or TSB medium. The $y$-axis of the chart shows the statistical significance. Each dot in the chart represents one annotated gene. Red dots: upregulated, green dots: downregulated, and blue dots: no significant change.

expression levels of seven other putative TonB-dependent transporters (B739_0115, B739_0876, B739_1045, B739_1343, B739_0216, B739_0329, and B739_0389) (Table S3) were downregulated in the presence of iron depletion. Upregulated TonB-dependent receptors would be predicted to be involved in iron or hemin uptake, while the functions of all downregulated TonB-dependent receptors are presently unknown. Similar results have been obtained in other bacteria, such as Pseudomonas fluorescens [28].

TonB-dependent receptors rely on the accessory proteins ExbB, ExbD, and TonB for energy transduction. One TonB family protein and two sets of ExbB-ExbD-TonB were found and identified in $R$. anatipestifer [14]. In other bacteria, such as E. coli [29] and Pseudomonas fluorescens [28], the ton $B$ gene is negatively regulated by iron. However, the transcription of ton $B$ genes in $R$. anatipestifer $\mathrm{CH}-1$ was not significantly changed in the iron-limited condition. To ensure the validity of the result, we also used qRT-PCR to measure ton $B$ gene transcription in iron-limited conditions. The result was coincident with that of RNA-Seq. These results suggested that, in contrast to many bacteria, the ton $B$ systems of $R$. anatipestifer $\mathrm{CH}-1$ are not regulated by iron.

Once siderophore-bound $\mathrm{Fe}^{3+}$ is transported into the cytoplasm, the iron must be released from the siderophore. 
TABLE 2: Genes upregulated in Riemerella anatipestifer $\mathrm{CH}-1$ in iron-depleted conditions.

\begin{tabular}{|c|c|c|c|c|}
\hline Gene ID & Gene name & log2.Fold_change. & $P$ value & Description \\
\hline 13715178 & B739_0074 & 1.289 & 0.00021641 & Hypothetical protein \\
\hline 13715197 & B739_0093 & 2.8509 & $2.72 E-108$ & Hypothetical protein \\
\hline 13715198 & B739_0094 & 1.2615 & $8.08 E-28$ & Outer membrane receptor for $\mathrm{Fe} 3+\mathrm{FecA}$ \\
\hline 13715199 & B739_0095 & 1.5902 & $5.31 E-23$ & Type I deoxyribonuclease HsdR \\
\hline 13715200 & B739_0096 & 3.3265 & $4.50 E-246$ & Hypothetical protein \\
\hline 13715201 & B739_0097 & 3.1172 & $1.67 E-115$ & Carbohydrate-binding protein \\
\hline 13715202 & B739_0098 & 3.3417 & 0 & Hypothetical protein \\
\hline 13715203 & B739_0099 & 3.5712 & $5.25 E-128$ & Hypothetical protein \\
\hline 13715204 & B739_0100 & 3.5135 & $5.06 E-149$ & Hypothetical protein \\
\hline 13715205 & B739_0101 & 3.0646 & $1.23 E-132$ & Substrate import-associated zinc metallohydrolase \\
\hline 13715206 & B739_0102 & 3.4075 & 0 & Glycan metabolism protein RagB \\
\hline 13715207 & B739_0103 & 3.8099 & 0 & TonB-linked outer membrane protein, SusC/RagA family \\
\hline 13715231 & B739_0127 & 1.275 & $1.56 E-06$ & DNA-binding protein \\
\hline 13715277 & B739_0173 & 3.3109 & $2.55 E-195$ & TonB-dependent receptor CirA, mostly Fe transport \\
\hline 13715278 & B739_0174 & 3.6434 & $5.92 E-216$ & Hypothetical protein \\
\hline 13715279 & B739_0175 & 2.413 & $5.96 E-53$ & Ankyrin \\
\hline 13715280 & B739_0176 & 3.0584 & $1.11 E-20$ & Predicted periplasmic protein \\
\hline 13715281 & B739_0177 & 1.0467 & $3.85 E-06$ & Nitric oxide synthase \\
\hline 13715373 & B739_2137 & 1.1196 & $6.02 E-07$ & $\begin{array}{l}\text { Camphor resistance protein CrcB; integral membrane } \\
\text { protein possibly involved in chromosome condensation } \\
\text { [cell division and chromosome partitioning] }\end{array}$ \\
\hline 13715432 & B739_0891 & 1.0093 & $1.05 E-06$ & Hypothetical protein \\
\hline 13715452 & B739_0912 & 1.0968 & $6.65 E-11$ & Ribonuclease III \\
\hline 13715453 & B739_0913 & 1.0147 & 0.0019348 & Hypothetical protein \\
\hline 13715513 & B739_0973 & 1.0814 & $1.74 E-42$ & 50S ribosomal protein L16/L10E \\
\hline 13715515 & B739_0975 & 1.0712 & $4.92 E-15$ & 30S ribosomal protein S17 \\
\hline 13715522 & B739_0982 & 1.1625 & $3.94 E-41$ & 50S ribosomal protein L18 \\
\hline 13715525 & B739_0985 & 1.2524 & $1.07 E-63$ & 50S ribosomal protein L15 \\
\hline 13715526 & B739_0986 & 1.0086 & $2.96 E-104$ & Preprotein translocase subunit SecY \\
\hline 13715542 & $-1 /-$ & 1.4285 & $1.38 E-29$ & tRNA-Glu \\
\hline 13715606 & B739_1068 & 3.8593 & 0 & FecA \\
\hline 13715627 & B739_1089 & 1.3526 & $1.76 E-65$ & Hypothetical protein \\
\hline 13715649 & B739_1112 & 1.0673 & $5.05 E-41$ & $50 S$ ribosomal protein $\mathrm{L} 31$ \\
\hline 13715783 & B739_1246 & 1.0718 & $1.24 E-91$ & 30S ribosomal protein S16 \\
\hline 13715836 & B739_1299 & 1.2361 & $5.75 E-09$ & Hypothetical protein \\
\hline 13715897 & B739_1360 & 1.3576 & 0.0002407 & Hypothetical protein \\
\hline 13715932 & B739_1395 & 1.4655 & $1.89 E-17$ & $\begin{array}{l}\text { 2-Amino-4-hydroxy-6-hydroxymethyldihydropteridine } \\
\text { pyrophosphokinase }\end{array}$ \\
\hline 13715934 & B739_1397 & 1.08 & $1.36 E-93$ & $\begin{array}{l}\text { Outer membrane protein-related } \\
\text { peptidoglycan-associated (lipo)protein }\end{array}$ \\
\hline 13715952 & B739_1415 & 4.7103 & 0 & Hypothetical protein \\
\hline 13715953 & B739_1416 & 3.7691 & 0 & FepA \\
\hline 13715954 & B739_1417 & 4.5479 & $5.15 E-244$ & HmuY \\
\hline 13715956 & B739_1419 & 1.2458 & $2.43 E-15$ & Restriction endonuclease $\mathrm{S}$ subunits, Hsds \\
\hline 13716000 & B739_1467 & 1.605 & $7.16 E-26$ & Hypothetical protein \\
\hline 13716023 & B739_1491 & 1.2534 & $2.55 E-67$ & Hypothetical protein \\
\hline
\end{tabular}


TABLE 2: Continued.

\begin{tabular}{|c|c|c|c|c|}
\hline Gene ID & Gene name & log2.Fold_change. & $P$ value & Description \\
\hline 13716028 & B739_1496 & 1.0255 & 0.00022217 & Hypothetical protein \\
\hline 13716038 & B739_1506 & 1.2051 & $1.41 E-13$ & Phosphate transport regulator \\
\hline 13716056 & B739_1525 & 1.0126 & $2.76 E-16$ & OmpA \\
\hline 13716068 & B739_1537 & 1.1923 & $2.42 E-35$ & Thioredoxin \\
\hline 13716179 & B739_1648 & 1.1687 & $1.56 E-06$ & Hypothetical protein \\
\hline 13716365 & B739_1842 & 1.4584 & $1.63 E-10$ & Hypothetical protein \\
\hline 13716406 & B739_1883 & 1.1398 & $2.85 E-14$ & Preprotein translocase subunit SecG \\
\hline 13716420 & B739_1898 & 1.1221 & $8.61 E-20$ & 3-Oxoacyl-(acyl-carrier-protein) synthase III \\
\hline 13716438 & B739_1916 & 1.1349 & $1.80 E-07$ & Hypothetical protein \\
\hline 13716459 & B739_1938 & 1.0469 & $7.76 E-115$ & Hypothetical protein \\
\hline 13716524 & B739_2003 & 1.1565 & $1.21 E-26$ & Polyisoprenoid-binding protein; YceI-like domain \\
\hline 13716533 & B739_2012 & 1.3031 & 0.0098864 & $\begin{array}{l}\text { Prevent-host-death protein; Antitoxin Phd_YefM, type II } \\
\text { toxin-antitoxin system }\end{array}$ \\
\hline 13716610 & B739_2089 & 1.0236 & 0.00048509 & Porin \\
\hline 13716613 & B739_2092 & 1.0406 & $3.79 E-32$ & Starch binding outer membrane protein SusD \\
\hline 13716712 & B739_0221 & 1.0457 & $3.80 E-21$ & Gliding motility protein GldL \\
\hline 13716745 & B739_0254 & 1.1712 & $1.78 E-48$ & Hypothetical protein \\
\hline 13716748 & B739_0257 & 1.0548 & $1.69 E-11$ & Hypothetical protein \\
\hline 13716800 & B739_0310 & 1.1729 & $4.55 E-21$ & Carbohydrate-binding protein SusD \\
\hline 13716803 & B739_0313 & 1.0601 & $2.33 E-59$ & Ribonuclease G \\
\hline 13716804 & B739_0314 & 1.3277 & $1.60 E-56$ & Bacterial nucleoid DNA-binding protein \\
\hline 13716824 & B739_0335 & 1.2846 & $9.72 E-16$ & Hypothetical protein \\
\hline 13716825 & B739_0336 & 1.0435 & $1.66 E-07$ & Hypothetical protein \\
\hline 13716826 & B739_0337 & 1.0406 & $3.10 E-06$ & Ras_like_GTPase \\
\hline 13716848 & B739_0360 & 1.2725 & $1.12 E-137$ & 50S ribosomal protein L11 \\
\hline 13716849 & B739_0361 & 1.0413 & $1.23 E-42$ & Transcription antiterminator \\
\hline 13716885 & B739_0397 & 1.0567 & $6.51 E-19$ & IMP dehydrogenase/GMP reductase \\
\hline 13716908 & B739_0420 & 1.0126 & $2.35 E-05$ & Sec-independent protein secretion pathway component \\
\hline 13716924 & B739_0436 & 1.019 & 0.00033736 & Predicted glycosyltransferases \\
\hline 13716964 & B739_0476 & 1.2682 & $6.15 E-21$ & Hypothetical protein \\
\hline 13716978 & B739_0490 & 2.3711 & $4.94 E-95$ & Ferritin-like domain \\
\hline 13717035 & B739_0547 & 1.2256 & $9.53 E-39$ & RNA polymerase Rpb6 \\
\hline 13717082 & B739_0594 & 1.082 & $1.16 E-21$ & Iron transporter FeoB \\
\hline 13717083 & B739_0595 & 2.259 & $5.94 E-27$ & Iron transporter FeoA \\
\hline 13717096 & B739_0608 & 1.6768 & $3.57 E-11$ & $\begin{array}{l}\text { Oxidoreductase; siderophore-interacting protein } \\
\text { [inorganic ion transport and metabolism]ViuB }\end{array}$ \\
\hline 13717113 & B739_0625 & 1.7097 & $2.66 E-21$ & RNA polymerase sigma factor \\
\hline 13717239 & B739_0753 & 1.1957 & $3.41 E-12$ & Transthyretin-like protein \\
\hline 13717245 & B739_0759 & 1.1112 & $2.64 E-14$ & Iron-sulfur binding protein \\
\hline
\end{tabular}

The first mechanism is that siderophore-bound Fe(III) is reduced to siderophore-bound Fe(II) followed by its spontaneous release due to the low affinity of iron $\mathrm{Fe}(\mathrm{II})$ with the siderophore. Another mechanism is that siderophorebound Fe(III) is hydrolyzed by specialized enzymes, leading to a dramatic loss of complex stability and facilitating the subsequent removal of the iron [30] in a reduction process. In this study, a gene coding for a siderophore-interacting protein (B739_0608) was upregulated significantly in the presence of iron depletion. This siderophore-interacting protein is involved in iron acquisition and virulence in $R$. anatipestifer strain CH-3 [31]. Surprisingly, among the upregulated genes, we did not find any homologue gene related to siderophore synthesis.

3.6. A Putative Polysaccharide Utilization Locus of R. anatipestifer CH-1 Was Upregulated in Iron-Limited Conditions. In Capnocytophaga canimorsus, a member of the Bacteroidetes, 


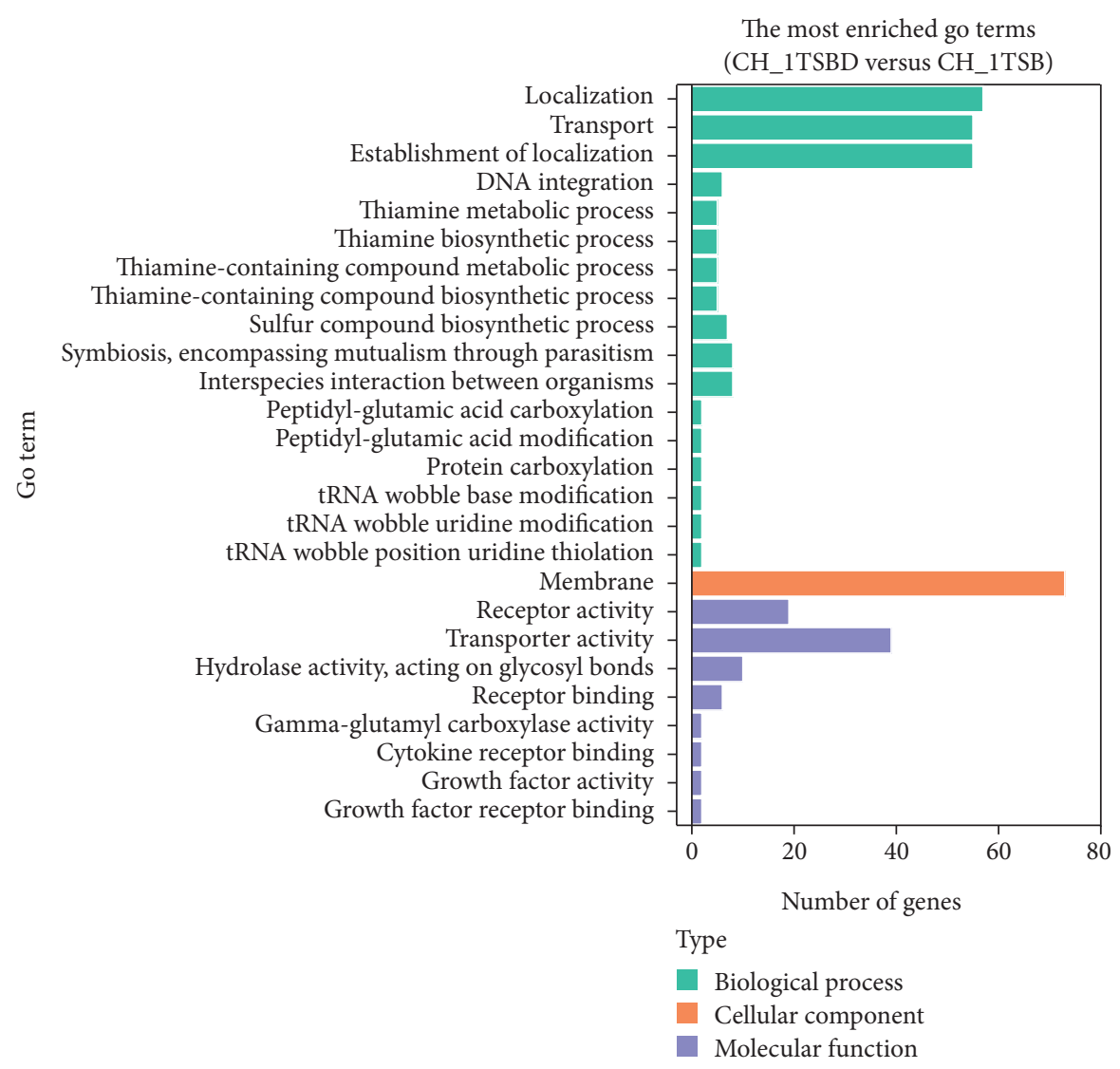

FIGURE 4: Role categories of genes from the transcriptome data. The numbers of genes that are up- and downregulated in $R$. anatipestifer $\mathrm{CH}-1$ grown in iron-limited TSB medium versus TSB medium are categorized according to role categories. Some genes are listed in more than one category and so may be counted more than once.

a polysaccharide utilization system uses serotransferrin as an iron source [32]. Each polypeptide encoded by this locus is required for this iron uptake activity [32]. This type of system was named the iron capture system (ICS), and it contains seven genes: $i c s A, i c s C, i c s D, i c s E, i c s F, i c s G$, and $i c s H$ [32]. In this study, we identified a gene cluster (B739_0094, B739_0095, B739_0096, B739_0097, B739_0098, B739_0099, B739_0100, B739_0101, B739_0102, and B739_0103) (Table 2) involved in polysaccharide utilization, the expression of which was upregulated in the presence of iron depletion. Sequence comparison showed that the homologues of $i c s C$, $i c s D$, icsE, icsF, ics $G$, and icsH from Capnocytophaga canimorsus are B739_0103, B739_0102, B739_0101, B739_0100, B739_0099, and B739_0098, respectively, in the R. anatipestifer CH-1 genome. The homologue of icsA, B739_1068, was not cotranscribed with the others. Interestingly, some genes that were upregulated in the gene cluster, such as B739_0094, B739_0095, B739_0096, and B739_0097, were not predicted to contribute to the ICS system. Additionally, the R. anatipestifer $\mathrm{CH}-1$ genome contains at least 6 polysaccharide utilization systems. In iron-limited conditions, three of the genes (locus B739_0094-B739_0103, locus B739_2091-B739_2093, and locus B739_0310-B739_0312) were upregulated (Table 2), while three other genes (locus B739_0115-B739_0118, locus B739_0875-B739_0876, and locus B739_1044-B739_1045) were downregulated (Table S3). Why some loci were upregulated and some loci were downregulated in the iron-limited condition is not currently understood.

\subsection{Iron Limitation Increased Transcription of Putative Hemin} Acquisition Systems. In the host, heme-containing proteins, such as hemoglobin, can be used as the main iron source by pathogenic bacteria [33]. Hemin uptake systems are regulated by iron in other bacteria $[34,35]$. Here, putative genes involved in hemin uptake were more highly expressed in iron-limited cultures of $R$. anatipestifer $\mathrm{CH}$ 1 than in iron-replete cultures. Within the upregulated genes, the most highly expressed gene cluster was FepAhmuY (B739_1416, B739_1417), which encodes a putative outer membrane ferrienterochelin, a colicin receptor and an HmuY-like hemophore protein. In Porphyromonas gingivalis, $\mathrm{HmuY}$ is a heme-binding lipoprotein associated with the outer membrane or secreted to the outside environment [36, 37]. Gene (B739_1415) adjacent to the FepA-hmuY operon was also upregulated in iron-limited medium. The functions of B739_1415, B739_1416, and B739_1417 in hemin utilization are underinvestigated in our group.

3.8. Transcription of Respiratory Chain Genes. In aerobic metabolism, the respiratory chain typically uses proteins that 


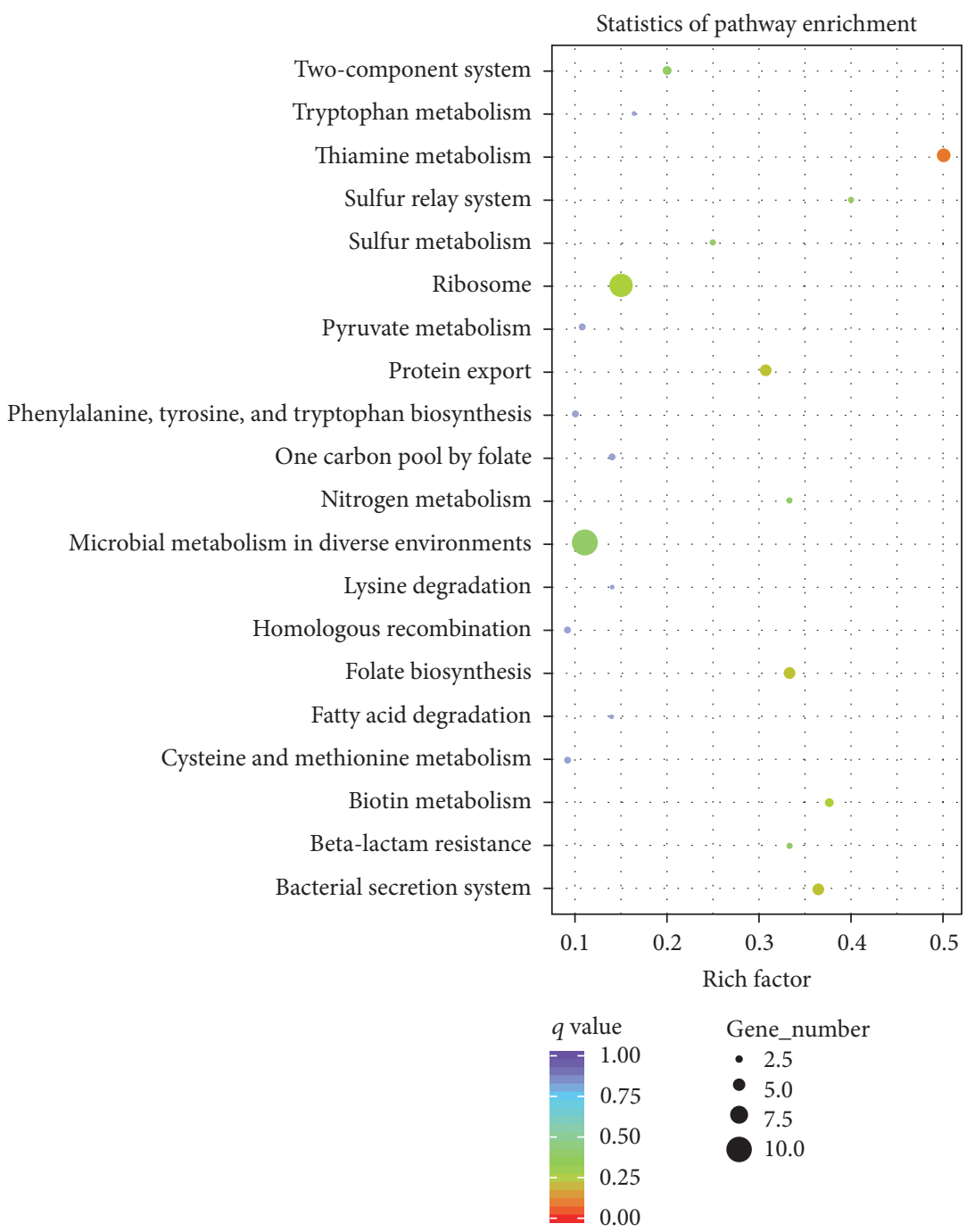

FIGURE 5: KEGG pathway enrichment analysis of differentially expressed genes between TSB and TSB with Dip. The $y$-axis of the chart shows the pathway name. The $x$-axis of the chart shows the Richness factor. The size of each point shows the number of genes in the pathway. The color of each point shows the $Q$ value range.

require iron as a cofactor [38]. When $R$. anatipestifer $\mathrm{CH}-1$ was grown in iron-limited medium, the expression of genes coding for cytochrome biogenesis protein (B739_0948), periplasmic cytochrome c552 subunit (B739_0946), and cytochrome C (B739_0186) were downregulated (Table S3). It indicated that iron restriction hindered $R$. anatipestifer aerobic metabolism. Similarly, in other bacteria, such as Pseudomonas fluorescens Pf- 5 , the transcription levels of genes encoding cytochrome c-type biogenesis proteins (PFL_168488) and subunits of cbb3-type cytochrome $c$ oxidases (PFL_1922-25, PFL_2834) are downregulated in iron-limited versus iron-replete medium [28].

3.9. Transcription of Genes Related to Natural Competence. Natural transformation refers to the process by which bacteria can actively take up and integrate exogenous DNA. Natural transformation is a major mechanism of horizontal gene transfer (HGT) and plays a prominent role in bacterial evolution [39]. The process of Vibrio cholerae natural transformation involves four steps: DNA-binding via type IV pili, DNA pulling via ComEA, DNA translocation via ComEC, and DNA recombination by the single-strand DNAbinding proteins DprA and RecA [40]. Previously, we found that $R$. anatipestifer $\mathrm{CH}-1$ is naturally competent [41]. In $R$. anatipestifer $\mathrm{CH}-1$, no putative type IV pilus locus is evident in the genome. In $R$. anatipestifer $\mathrm{CH}-1$, two proteins that are predicted to be involved in the DNA uptake process, a ComEC homologue (B739_1095) and a gene encoding a single-strand DNA-binding protein (B739_1757), were downregulated in iron-limited conditions. One possibility for this phenomenon is that these proteins require iron for activity, as well as iron being predicted to be involved in the natural transformation process. This relationship between natural transformation and iron availability has not yet been described. 


\section{Conclusion}

In this study, we examined the transcriptomic impact of iron limitation on $R$. anatipestifer $\mathrm{CH}-1$ by comparing ironlimited TSB cultures with iron-replete TSB cultures. This transcriptome analysis identified numerous genes involved in $R$. anatipestifer $\mathrm{CH}-1$ iron utilization. Under iron limitation, we observed changes in the transcription levels of genes related to iron homeostasis functions, such as the Feo system, the ICS system, and other iron uptake systems. Iron limitation also resulted in several unexpected responses, particularly the increased transcription of the ribosomal protein genes L18, L15, and L31. The data in this study were useful for identifying genes involved in iron utilization in $R$. anatipestifer $\mathrm{CH}$ 1 and for shedding light on the adaptation mechanisms of R. anatipestifer CH-1 in iron-limited environments, such as hosts.

$\begin{array}{ll}\text { Abbreviations } \\ \text { RNA-Seq: } & \text { RNA sequencing } \\ \text { qRT-PCR: } & \begin{array}{l}\text { Quantitative reverse transcription } \\ \text { polymerase chain reaction }\end{array} \\ \text { HGT: } & \text { Horizontal gene transfer } \\ \text { DEGs: } & \text { Differentially expressed genes } \\ \text { KEGG: } & \text { Kyoto encyclopedia of genes and genomes } \\ \text { GO: } & \text { Gene Ontology } \\ \text { ICS: } & \text { Iron capture system } \\ \text { FPKM: } & \text { Expected number of Fragments Per } \\ & \text { Kilobase of transcript sequence per } \\ & \text { Million base pairs sequenced. }\end{array}$

\section{Conflicts of Interest}

The authors declare that they have no competing interests.

\section{Authors' Contributions}

MaFeng Liu and AnChun Cheng conceived and designed the experiments. MaFeng Liu and Mi Huang performed the experiments. Francis Biville, MingShu Wang, DeKang Zhu, RenYong Jia, Shun Chen, and KunFeng Sun analyzed the data. Ying $\mathrm{Wu}$ and Qiao Yang contributed reagents/materials/analysis tools. MaFeng Liu and Mi Huang wrote the paper. All authors have reviewed the manuscript. MaFeng Liu and Mi Huang contributed equally to this work.

\section{Acknowledgments}

This work was supported by the International S\&T Cooperation Program of Sichuan Province (Grant no. 2016HH0052), the National Natural Science Foundation of China (Grant no. 31302131, http://www.nsfc.gov.cn/), the Research Fund for the Doctoral Program of Higher Education of China (Grant no. 20135103120006, http://www.cutech.edu.cn/cn/index.htm), the National Science and Technology Support Program (no. 2015BAD12B05), the China Agricultural Research System (CARS-43-8), and the Integration and Demonstration of Key
Technologies for Duck Industrialization in Sichuan Province (2014NZ0030).

\section{References}

[1] S. Subramaniam, K.-L. Chua, H.-M. Tan, H. Loh, P. Kuhnert, and J. Frey, "Phylogenetic position of Riemerella anatipestifer based on 16S rRNA gene sequences," International Journal of Systematic Bacteriology, vol. 47, no. 2, pp. 562-565, 1997.

[2] S. Subramaniam, B. Huang, H. Loh et al., "Characterization of a predominant immunogenic outer membrane protein of Riemerella anatipestifer," Clinical and Diagnostic Laboratory Immunology, vol. 7, no. 2, pp. 168-174, 2000.

[3] S. Leavitt and M. Ayroud, "Riemerella anatipestifer infection of domestic ducklings," Canadian Veterinary Journal, vol. 38, no. 2, p. 113, 1997.

[4] P. Pathanasophon, P. Phuektes, T. Tanticharoenyos, W. Narongsak, and T. Sawada, "A potential new serotype of Riemerella anatipestifer isolated from ducks in Thailand," Avian Pathology, vol. 31, no. 3, pp. 267-270, 2002.

[5] C. Wandersman and P. Delepelaire, "Bacterial iron sources: From siderophores to hemophores," Annual Review of Microbiology, vol. 58, pp. 611-647, 2004.

[6] L. J. Runyen-Janecky, "Role and regulation of heme iron acquisition in gram-negative pathogens," Frontiers in Cellular and Infection Microbiology, vol. 4, article 55, 2013.

[7] S. C. Andrews, A. K. Robinson, and F. Rodríguez-Quiñones, "Bacterial iron homeostasis," FEMS Microbiology Reviews, vol. 27, no. 2-3, pp. 215-237, 2003.

[8] R. C. Hider and X. Kong, "Chemistry and biology of siderophores," Natural Product Reports, vol. 27, no. 5, pp. 637$657,2010$.

[9] N. Noinaj, M. Guillier, T. J. Barnard, and S. K. Buchanan, “TonBdependent transporters: regulation, structure, and function," Annual Review of Microbiology, vol. 64, pp. 43-60, 2010.

[10] S. Cescau, H. Cwerman, S. Létoffé, P. Delepelaire, C. Wandersman, and F. Biville, "Heme acquisition by hemophores," BioMetals, vol. 20, no. 3-4, pp. 603-613, 2007.

[11] J. P. McHugh, F. Rodríguez-Quiñones, H. Abdul-Tehrani et al., "Global iron-dependent gene regulation in Escherichia coli: a new mechanism for iron homeostasis," Journal of Biological Chemistry, vol. 278, no. 32, pp. 29478-29486, 2003.

[12] C. Yu, R. McClure, K. Nudela, N. Daou, and C. A. Genco, "Characterization of the Neisseria gonorrhoeae Iron and Fur regulatory network," Journal of Bacteriology, vol. 198, no. 16, pp. 2180-2191, 2016.

[13] J. Butcher and A. Stintzi, "The transcriptional landscape of Campylobacter jejuni under iron replete and iron limited growth conditions," PLoS ONE, vol. 8, no. 11, Article ID e79475, 2013.

[14] H. Liao, X. Cheng, D. Zhu et al., “TonB energy transduction systems of riemerella anatipestifer are required for iron and hemin utilization," PLoS ONE, vol. 10, no. 5, Article ID e0127506, 2015.

[15] X. Wang, W. Liu, D. Zhu et al., "Comparative genomics of Riemerella anatipestifer reveals genetic diversity," $B M C$ Genomics, vol. 15, no. 1, article 479, 2014.

[16] H. Liao, M. Liu, X. Cheng et al., "The detection of heminbinding proteins in riemerella anatipestifer $\mathrm{CH}-1$," Current Microbiology, vol. 72, no. 2, pp. 152-158, 2016. 
[17] M. Liu, M. Wang, D. Zhu et al., "Investigation of TbfA in Riemerella anatipestifer using plasmid-based methods for gene over-expression and knockdown," Scientific Reports, vol. 6, p. 37159, 2016.

[18] J. Liu, S. Wang, T. Qin et al., "Whole transcriptome analysis of Penicillium digitatum strains treatmented with prochloraz reveals their drug-resistant mechanisms," BMC Genomics, vol. 16, no. 1, article 855, 2015.

[19] B. Langmead and S. L. Salzberg, "Fast gapped-read alignment with Bowtie 2," Nature Methods, vol. 9, no. 4, pp. 357-359, 2012.

[20] S. Anders, P. T. Pyl, and W. Huber, "HTSeq-a Python framework to work with high-throughput sequencing data," Bioinformatics, vol. 31, no. 2, pp. 166-169, 2015.

[21] M. D. Robinson, D. J. McCarthy, and G. K. Smyth, "edgeR: a Bioconductor package for differential expression analysis of digital gene expression data," Bioinformatics, vol. 26, no. 1, pp. 139-140, 2010.

[22] S. Anders and W. Huber, "Differential expression analysis for sequence count data," Genome Biology, vol. 11, no. 10, article R106, 2010.

[23] R. McClure, D. Balasubramanian, Y. Sun et al., "Computational analysis of bacterial RNA-Seq data," Nucleic Acids Research, vol. 41, no. 14, article e140, 2013.

[24] X. Mao, T. Cai, J. G. Olyarchuk, and L. Wei, "Automated genome annotation and pathway identification using the KEGG Orthology (KO) as a controlled vocabulary," Bioinformatics, vol. 21, no. 19, pp. 3787-3793, 2005.

[25] C. K. Y. Lau, K. D. Krewulak, and H. J. Vogel, "Bacterial ferrous iron transport: the Feo system," FEMS Microbiology Reviews, vol. 40, no. 2, Article ID fuv049, pp. 273-298, 2016.

[26] S. W. Bearden and R. D. Perry, "The Yfe system of Yersinia pestis transports iron and manganese and is required for full virulence of plague," Molecular Microbiology, vol. 32, no. 2, pp. 403-414, 1999.

[27] J. Cao, M. R. Woodhall, J. Alvarez, M. L. Cartron, and S. C. Andrews, "EfeUOB (YcdNOB) is a tripartite, acid-induced and CpxAR-regulated, low-pH Fe2+ transporter that is cryptic in Escherichia coli K-12 but functional in E. coli O157:H7," Molecular Microbiology, vol. 65, no. 4, pp. 857-875, 2007.

[28] C. K. Lim, K. A. Hassan, S. G. Tetu, J. E. Loper, and I. T. Paulsen, "The effect of iron limitation on the transcriptome and proteome of Pseudomonas fluorescens Pf-5," PLoS ONE, vol. 7, no. 6, Article ID e39139, 2012.

[29] P. I. Higgs, R. A. Larsen, and K. Postle, "Quantification of known components of the Escherichia coli TonB energy transduction system: TonB, ExbB, ExbD and FepA," Molecular Microbiology, vol. 44, no. 1, pp. 271-281, 2002.

[30] M. Miethke and M. A. Marahiel, "Siderophore-based iron acquisition and pathogen control," Microbiology and Molecular Biology Reviews, vol. 71, no. 3, pp. 413-451, 2007.

[31] J. Tu, F. Lu, S. Miao et al., "The siderophore-interacting protein is involved in iron acquisition and virulence of riemerella anatipestifer strain CH3," Veterinary Microbiology, vol. 168, no. 2-4, pp. 395-402, 2014.

[32] P. Manfredi, F. Lauber, F. Renzi, K. Hack, E. Hess, and G. R. Cornelis, "New iron acquisition system in Bacteroidetes," Infection and Immunity, vol. 83, no. 1, pp. 300-310, 2015.

[33] T. A. Rouault, "Pathogenic bacteria prefer heme," Science, vol. 305, no. 5690, pp. 1577-1578, 2004.

[34] H. Contreras, N. Chim, A. Credali, and C. W. Goulding, "Heme uptake in bacterial pathogens," Current Opinion in Chemical Biology, vol. 19, no. 1, pp. 34-41, 2014.
[35] J. R. Sheldon and D. E. Heinrichs, "Recent developments in understanding the iron acquisition strategies of gram positive pathogens," FEMS Microbiology Reviews, vol. 39, no. 4, pp. 592630, 2015.

[36] T. Olczak, A. Sroka, J. Potempa, and M. Olczak, "Porphyromonas gingivalis $\mathrm{HmuY}$ and $\mathrm{HmuR}$ : further characterization of a novel mechanism of heme utilization," Archives of Microbiology, vol. 189, no. 3, pp. 197-210, 2008.

[37] H. Wojtowicz, T. Guevara, C. Tallant et al., "Unique structure and stability of HmuY, a novel heme-binding protein of Porphyromonas gingivalis," PLoS Pathogens, vol. 5, no. 5, p. e1000419, 2009.

[38] U. A. Ochsner, P. J. Wilderman, A. I. Vasil, and M. L. Vasil, "GeneChip ${ }^{\circledR}$ expression analysis of the iron starvation response in Pseudomonas aeruginosa: identification of novel pyoverdine biosynthesis genes," Molecular Microbiology, vol. 45, no. 5, pp. 1277-1287, 2002.

[39] J. P. Gogarten and J. P. Townsend, "Horizontal gene transfer, genome innovation and evolution," Nature Reviews Microbiology, vol. 3, no. 9, pp. 679-687, 2005.

[40] N. Matthey and M. Blokesch, "The DNA-uptake process of Naturally Competent Vibrio cholerae," Trends in Microbiology, vol. 24, no. 2, pp. 98-110, 2016.

[41] M. Liu, L. Zhang, L. Huang et al., "Use of Natural Transformation To Establish an Easy Knockout Method in Riemerella anatipestifer," Applied and Environmental Microbiology, vol. 83, no. 9, 2017. 

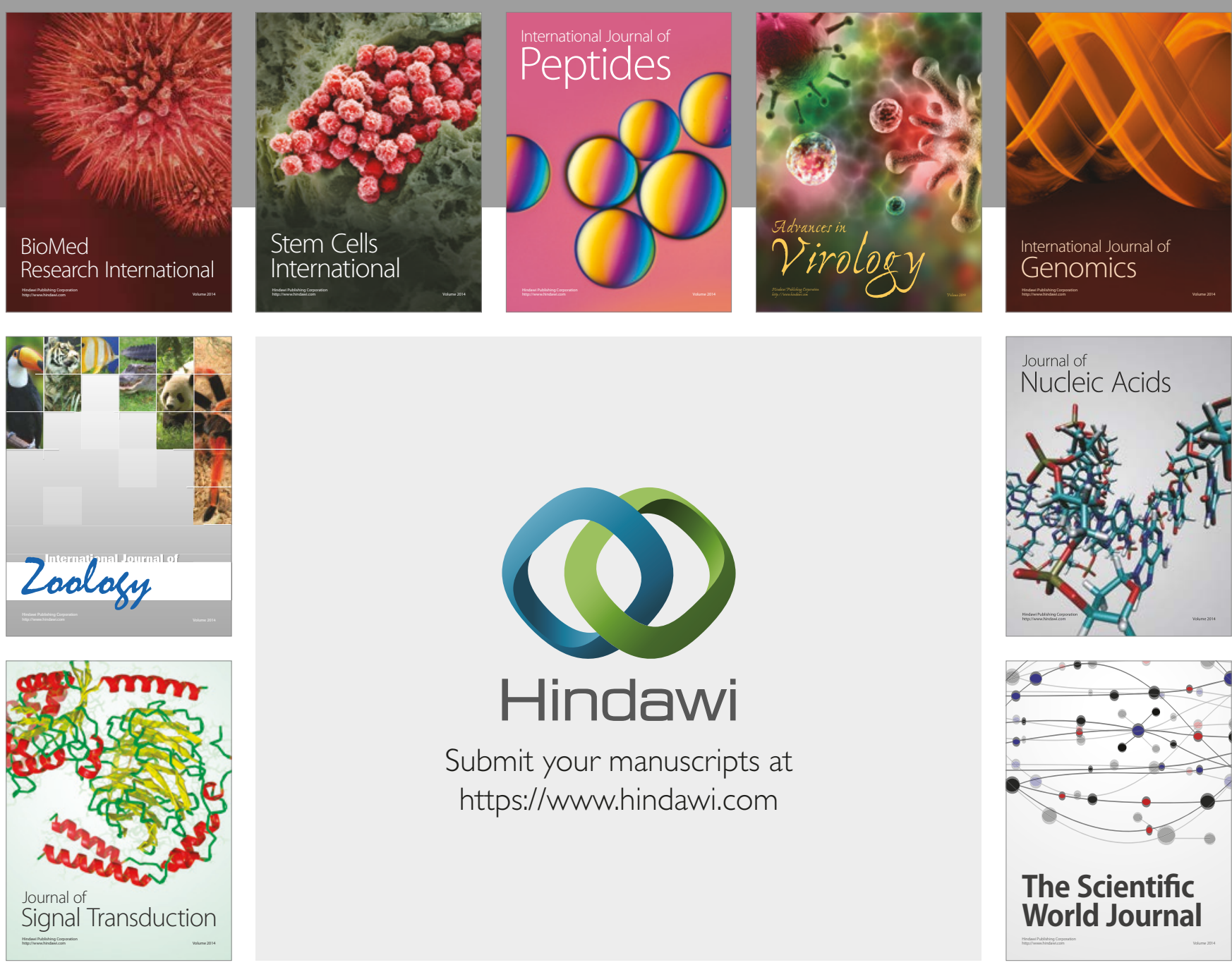

Submit your manuscripts at

https://www.hindawi.com
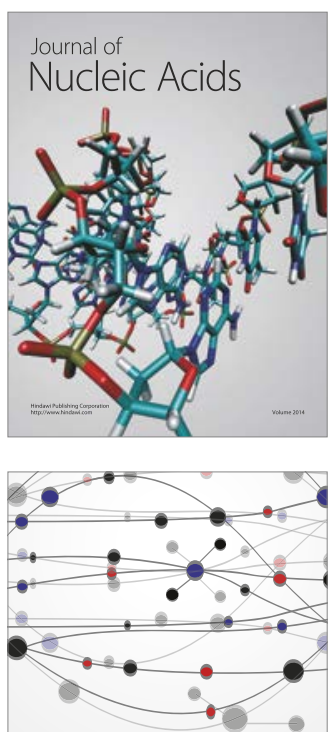

The Scientific World Journal

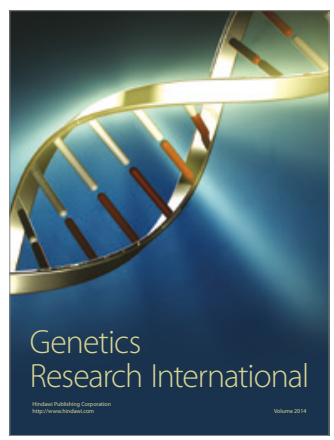

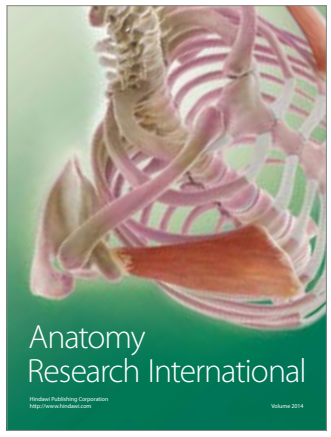

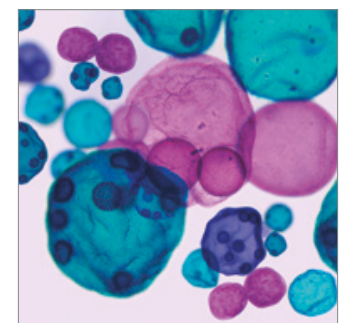

International Journal of Microbiology
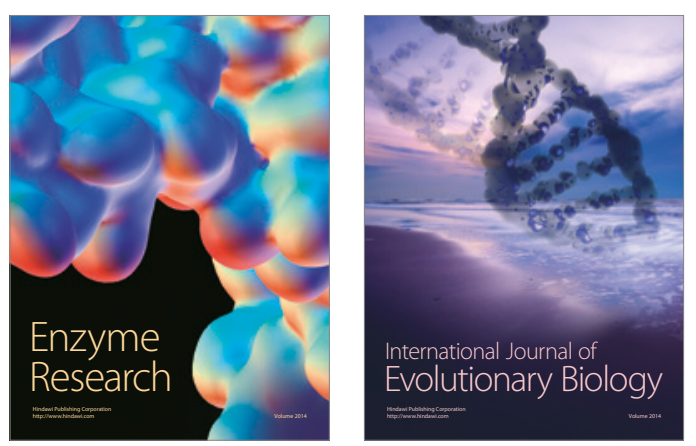
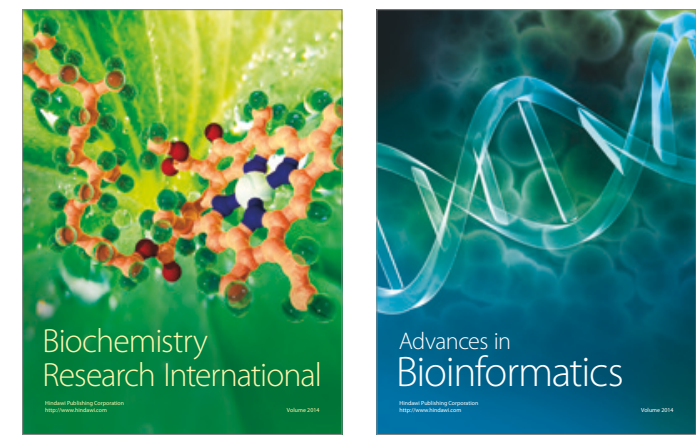

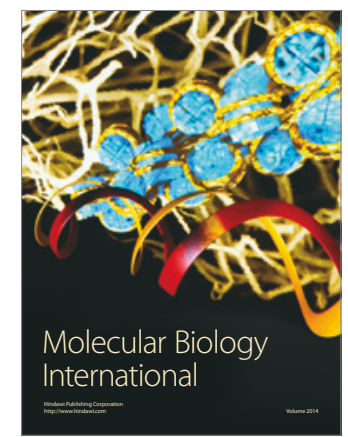

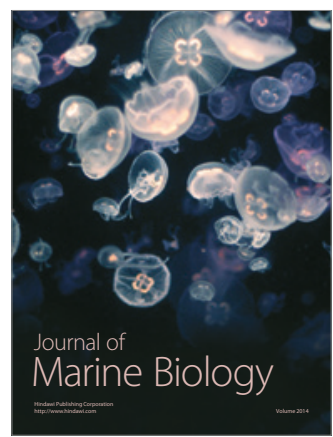

